\title{
Bayesian Analysis in Plant Pathology
}

\author{
A. L. Mila and A. L. Carriquiry
}

First author: Department of Plant Pathology, University of California-Davis, Kearney Agricultural Center, Parlier 93648; and second author: Department of Statistics, Iowa State University, Ames 50011.

Accepted for publication 16 May 2004.

\begin{abstract}
Mila, A. L., and Carriquiry, A. L. 2004. Bayesian analysis in plant pathology. Phytopathology 94:1027-1030.

Bayesian methods are currently much discussed and applied in several disciplines from molecular biology to engineering. Bayesian inference is the process of fitting a probability model to a set of data and summarizing

the results via probability distributions on the parameters of the model and unobserved quantities such as predictions for new observations. In this paper, after a short introduction of Bayesian inference, we present the basic features of Bayesian methodology using examples from sequencing genomic fragments and analyzing microarray gene-expressing levels, reconstructing disease maps, and designing experiments.
\end{abstract}

An important goal in statistical analyses is to draw inference about unobserved quantities. Within a classical statistics framework, inferences often take the form of point estimates of unknown parameters, and uncertainty about the true parameter value is represented with confidence intervals (7). In contrast, Bayesian statisticians make use of probability theory to reflect uncertainty; within the Bayesian framework, parameters are treated as if they were random variables, and described with probability distributions rather than point estimates (4).

In Bayesian analysis, there are three key components associated with parameter estimation: prior distribution, likelihood function, and posterior distribution. These three components are formally combined by Bayes' rule:

$$
\begin{array}{ccccc}
f\left(\theta \mid y_{\mathrm{obs}}\right) & \propto & g(\theta) & \times & L\left(y_{\mathrm{obs}} \mid \theta\right) \\
\text { posterior } & & \text { prior } & & \text { likelihood } \\
\text { distribution } & & \text { distribution } & & \text { function }
\end{array}
$$

where the symbol $\propto$ denotes proportionality, and $\theta$ is a set of parameters to be estimated (4). Sample data, $y_{\mathrm{obs}}$, are used to construct the likelihood function. Existing information about the parameters of interest, $\theta$, can be summarized quantitatively via probability distributions called the prior distributions of the parameters of interest. Depending on the level of existing evidence, a prior distribution may be noninformative (or vague) when no evidence is available, informative when some evidence is available, or very informative when strong evidence is available (4).

In the past, the use of probabilities to express prior belief has often been thought of as a feature of Bayesian inference and not as a necessity for all scientific inference. This was derived from the idea that model assumptions do not count as a prior belief (1). However, in linear regression, for example, parameter estimation depends heavily on the assumption of normality and, thus, regression may be imagined as embedded in a more complex model. From this viewpoint, classical statistical theory is not free from

Corresponding author: A. L. Mila; E-mail address: mmila@uckac.edu

Publication no. P-2004-0719-070

(C) 2004 The American Phytopathological Society assumptions of prior knowledge and shares more common ground with Bayesian theory than once was believed (1).

Despite the simple form of equation 1, there are two challenging aspects associated with Bayesian statistics: (i) Development of a model, $g(\theta) \times L\left(y_{\text {obs }} \mid \theta\right)$, which captures effectively the features of the scientific problem, and (ii) complex computation for deriving the posterior distribution (7). Until recently, computation was often difficult so that Bayesian analysis was mainly restricted to specialists. The introduction of iterative simulation methods, such as Markov Chain Monte Carlo techniques facilitated the broad application of Bayesian methods (7).

Features of Bayesian analysis. Use of probabilities to describe uncertainty. Uncertainty is an unavoidable part of life. In classical statistics, uncertainty is described by frequency, i.e., the frequency of observing the same outcome when the experiment is repeated many times. In Bayesian statistics, uncertainty is described by probabilities $(4,6)$. To understand this fundamental difference between classical and Bayesian statistics, consider the case of $95 \%$ confidence intervals $\left(\mathrm{CI}_{95}\right)$ in parameter estimation. In normal linear regression, uncertainty about a parameter estimate, $\hat{a}$, is summarized by a $\mathrm{CI}_{95}$ of the general form $(\hat{a}-1.96 \mathrm{SE}, \hat{a}+$ $1.96 \mathrm{SE})$, where $\mathrm{SE}$ is the standard error of the estimate. The interpretation in classical statistics is the following: 95 out of 100 times the (random) confidence interval will contain the (fixed) true parameter $\alpha$. Within the Bayesian framework, a normal 95\% confidence interval (also called posterior credible sets, CS) would be constructed in the same manner but its interpretation would be rather different. Bayesian statistics state that there is a $95 \%$ probability that the unknown $\alpha$ lies in the fixed CS. More generally, a $95 \%$ posterior $\mathrm{CS}$ is the set $(\mathrm{L}, \mathrm{U})$, where $\mathrm{L}$ and $\mathrm{U}$ denote the 2.5 th and the 97.5th percentiles of the posterior distribution of the parameter, whether the distribution is normal or not. Only in the case that the distribution is normal do the $95 \% \mathrm{CI}$ and the $95 \% \mathrm{CS}$ coincide.

Another example is given in Figure 1 (A. L. Mila, A. L. Carriquiry, and X. B. Yang, unpublished data). Bayesian methods were applied to account for uncertainty in prediction of Sclerotinia stem rot (SSR) prevalence in four states of the North-Central region of the United States. Figure 1 summarizes the predicted prevalence of SSR in northwestern Ohio in 1996. The histogram was constructed using 1,000 draws from the posterior distribution 
(SSR prevalence) that was based on a likelihood function constructed from data collected between 1995 and 1998 and noninformative prior distributions for the parameters (i.e., explanatory variables). Prior distributions and likelihood function were combined (equation 1) to generate posterior distributions such as the one in Figure 1. There is a $69 \%$ chance that SSR prevalence in northwestern Ohio is between 0.05 and 0.11 (i.e., $100 \times(215+$ $265+210) / 1,000=69 \%$ ) and there is only a $0.5 \%$ chance that SSR prevalence is higher than 0.19 (Fig. 1).

Combine information (priors and current data). The Bayesian approach lends itself very naturally to situations in which we wish to introduce prior information into our analysis of experimental data. This feature will be demonstrated with two examples from the field of bioinformatics: sequence segmentation and Bayesian analysis of gene expression levels. In sequence segmentation, we are given only the complete sequence, $R=\left(r_{1}, \ldots r_{j}\right)$, where $r_{j}=\{\mathrm{A}, \mathrm{G}, \mathrm{C}, \mathrm{T}\}$ but we do not know the exact composition of each position, nor do we know the number and location of change points, $A$, in the DNA sequence of interest (7).

Assume that the probability of A to be in position 3 is $P_{3}(\mathrm{~A})=$ $\theta_{\mathrm{A} 3}$, and there are four such probabilities, one for each base, $\Theta_{3}=$ $\left(\theta_{\mathrm{A} 3}, \theta_{\mathrm{T} 3}, \theta_{\mathrm{G} 3}, \theta_{\mathrm{C} 3}\right)$. We might be interested in drawing inferences for the number of change points, $A$, the probability that a change point will occur at position $v$ of the sequence, and the composition, $\Theta$, of each position of the fragment with Bayes' rule:

$$
P(A, \Theta, k \mid R)=P(R \mid A, \Theta) P(k \mid A) P(A) P(\Theta)
$$

$$
\text { Posterior Likelihood Priors }
$$

Here, $R$ denotes the complete sequence with $k$ segments of the same base. We need to assign prior distributions on the number of change points, $A$, and composition, $\Theta$, i.e., $P(A)$ and $P(\Theta)$, respectively, in equation 2. Commonly, these prior distributions derive from known DNA sequences of close relative species or strains.

Figures 2 to 3 present the posterior probability distributions of the number of change points, change point positions, and segmented composition for a fragment of the DNA sequence of Saccharomyces cerevisiae, the 500 bp upstream of the translational start site of the histone $H 1$ gene $(H 1: 500 b p)$. Results suggest the presence of three to five change points in the $H 1$ gene (Fig. 2), most likely around 230, 260, 337, and 460 bp (Fig. 3). The probability of each base to be in a position $v$ of the sequence changes significantly around the base pairs where change points may also be located (Fig. 3).

For the second example, assume that a culture of yeast is exposed during log-phase growth to a 30-min ethanol shock and then the level of gene expression of the ethanol-exposed cells is compared with the level of gene expression in the unexposed cells using microarray technology (11). Commonly, in interpretation of

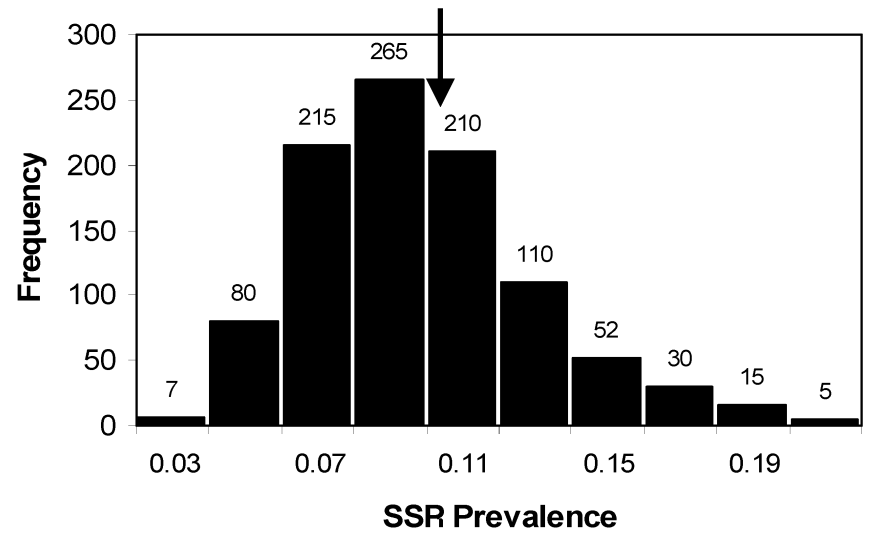

Fig. 1. Frequency of 1,000 posterior draws of prediction of Sclerotinia stem rot (SSR) prevalence, caused by Sclerotinia sclerotiorum, in northwestern Ohio in 1996. The arrow represents the observed prevalence in this area in 1996.
cDNA microarray data, the ratio, $z$, of expression levels of two samples, $i$ and $j$, is used instead of the absolute values. The ratio $z$ is considered to be drawn from $N\left(\mu_{i}, \sigma_{i}^{2}\right) / N\left(\mu_{j}, \sigma_{j}^{2}\right)$, i.e., the ratio of two normal distributions for samples $i$ and $j$. Thus, the gene expression level for each sample is assumed to follow a normal distribution with mean, $\mu$, and variance, $\sigma^{2}$. Using Bayes' rule, we combine observed ratio data, $f\left(z \mid \mu_{i}, \mu_{j}, \sigma_{i}^{2}, \sigma_{j}^{2}\right)$, with prior information for the means $\mu_{i}$ and $\mu_{j}$ and the variances $\sigma_{i}^{2}$ and $\sigma_{j}^{2}, g\left(\mu_{i}\right.$, $\left.\mu_{j}, \sigma_{i}^{2}, \sigma_{j}^{2}\right)$, to draw inference about the unknowns $\mu_{i}, \mu_{j}, \sigma_{i}^{2}$, and $\sigma_{j}^{2}: h\left(\mu_{i}, \mu_{j}, \sigma_{i}^{2}, \sigma_{j}^{2} \mid z\right) \propto f\left(z \mid \mu_{i}, \mu_{j}, \sigma_{i}^{2}, \sigma_{j}^{2}\right) \times g\left(\mu_{i}, \mu_{j}, \sigma_{i}^{2}, \sigma_{j}^{2}\right)$. Based on hypothetical data, Figure 4 illustrates one of the challenges that arise in the analysis of microarray data. It also highlights a feature of Bayesian analysis that can be viewed both as a virtue and a drawback. If, in the model above, noninformative priors are chosen for the two variance components, the posterior credible set of the gene expression ratios for ethanol-exposed and non-ethanol-exposed yeast overlap greatly (Fig. 4). A practitioner would conclude that there is no effect of ethanol treatment on the particular gene expression. However, an informative prior on the variances produces credible sets that do not overlap, suggesting a strong effect of ethanol shock on the expression of this particular gene (Fig. 4). The prior distribution can have a noticeable effect on the posterior distribution of some parameters when the likelihood does not contain "enough" information about the parameters of interest. If reliable prior information is available, then the Bayesian is at an advantage over the classical statistician, who typically relies only on sample data (11).

Add complexity in model structure. This feature will be demonstrated with an example from disease map reconstruction. Analysis of the geographic distribution of disease incidence or prevalence is important for public health workers and human epidemiologists (5). A related issue in disease map reconstruction is the reduction of noise in a disease map (5). The simplest possible mapping form is the depiction of crude disease rates at specific locations (e.g., individual fields, counties, and arbitrarily divided regions). An example from human epidemiology is shown in Figure 5 (12). The subregions are the zip codes as defined in 1983 for San Diego County, California. In the first panel (Fig. 5A), crude rates of hospitalization of children due to asthma are shown.

Interpretation of the structure of these events is limited by two factors. There is no information about the spatial distribution of the population that might be at risk of contracting the disease of concern, which also gave rise to the crude rates pictured below, and failure to account for this spatial distribution of the overall population may limit the interpretation of the generated map. For example, zip regions with low population density may yield high incidence rates even when just a few cases are observed. Thus, without accounting for this difference in population distribution, some areas could be incorrectly assigned a high disease risk (5).

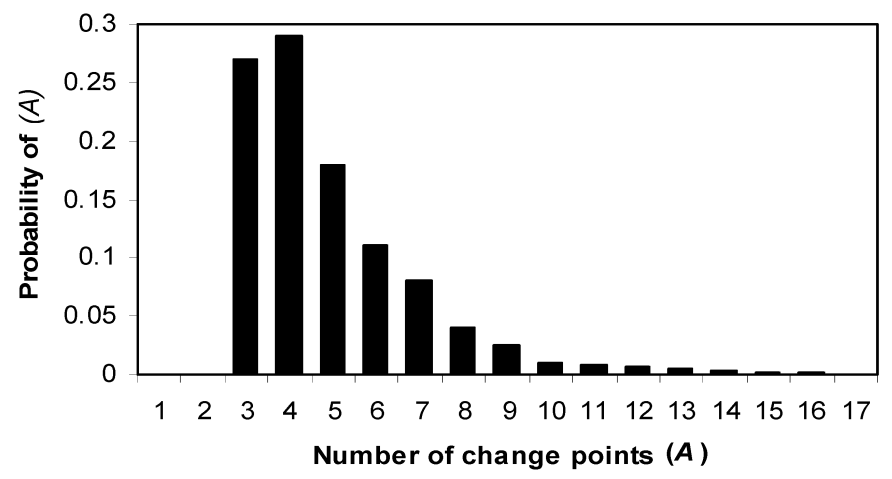

Fig. 2. Posterior probability distribution of the number of change points $(A)$ for the $500 \mathrm{bp}$ upstream of the translational start site of the histone gene $\mathrm{Hl}$ in Saccharomyces cerevisiae. (reproduced, with permission, from Liu and Lawrence [7]; copyright Oxford University Press). 
Depiction of a crude rates map is a helpful preliminary approach, but in a later stage, disease map construction is more informative if it is performed by introducing some model structure or constraints into the mapping process. There are many classical and Bayesian model-based approaches for map reconstruction. Here, we describe a three-stage Bayesian hierarchical model, which is often applied in human epidemiology to analyze data for disease mapping $(8,12)$.

If $Y_{j}$ is disease incidence in the form of disease counts in area $j$ and the disease is rare and noninfectious, we may assume

$$
Y_{j} \sim \operatorname{Poisson}\left(p_{j}\right)
$$

where $p_{j}$ is the risk of disease incidence in area $j$. Risk is assumed to be constant within an area. Model 3 is the first level in the hierarchy. In the second level, area risks, $p_{j}$, are modeled as

$$
\log \left(p_{j}\right)=X_{j}^{T} \beta^{T}+V_{j}+U_{j}
$$

where $X_{j}^{T}=\left(X_{j 1}, \ldots X_{j k}\right)$ is a $k \times 1$ vector of area-level risk covariates, $\beta^{T}=\left(\beta_{1}, \ldots \beta_{k}\right)$ is a $k \times 1$ vector of unknown regression parameters, $V_{j}$ represents a residual with no spatial structure (so that $V_{i}$ and $V_{j}$ are independent for $i \neq j$ ), and $U_{j}$ represents a residual with spatial structure (e.g., $U_{i}$ and $U_{j}$ are modeled to have positive spatial dependence) (8). Rates $p_{j}$ are modeled in the log scale to ensure that $p_{j}$ remains positive, even though a linear regression relation is chosen to include the covariates.

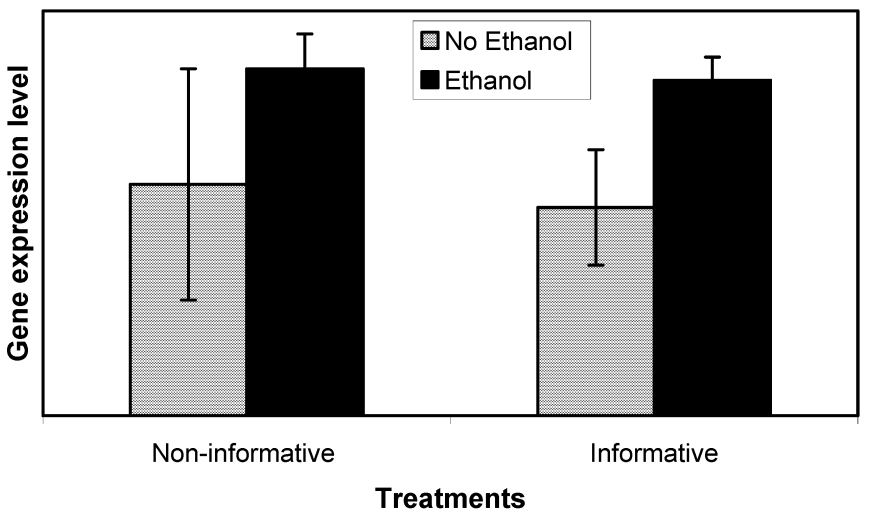

Fig. 4. Estimated means of gene expression level for yeast cells that were exposed or not exposed to a 30-min ethanol shock. Noninformative and informative prior distributions for the variances of gene expression levels were used. Bars represent posterior confidence intervals of gene expression levels.
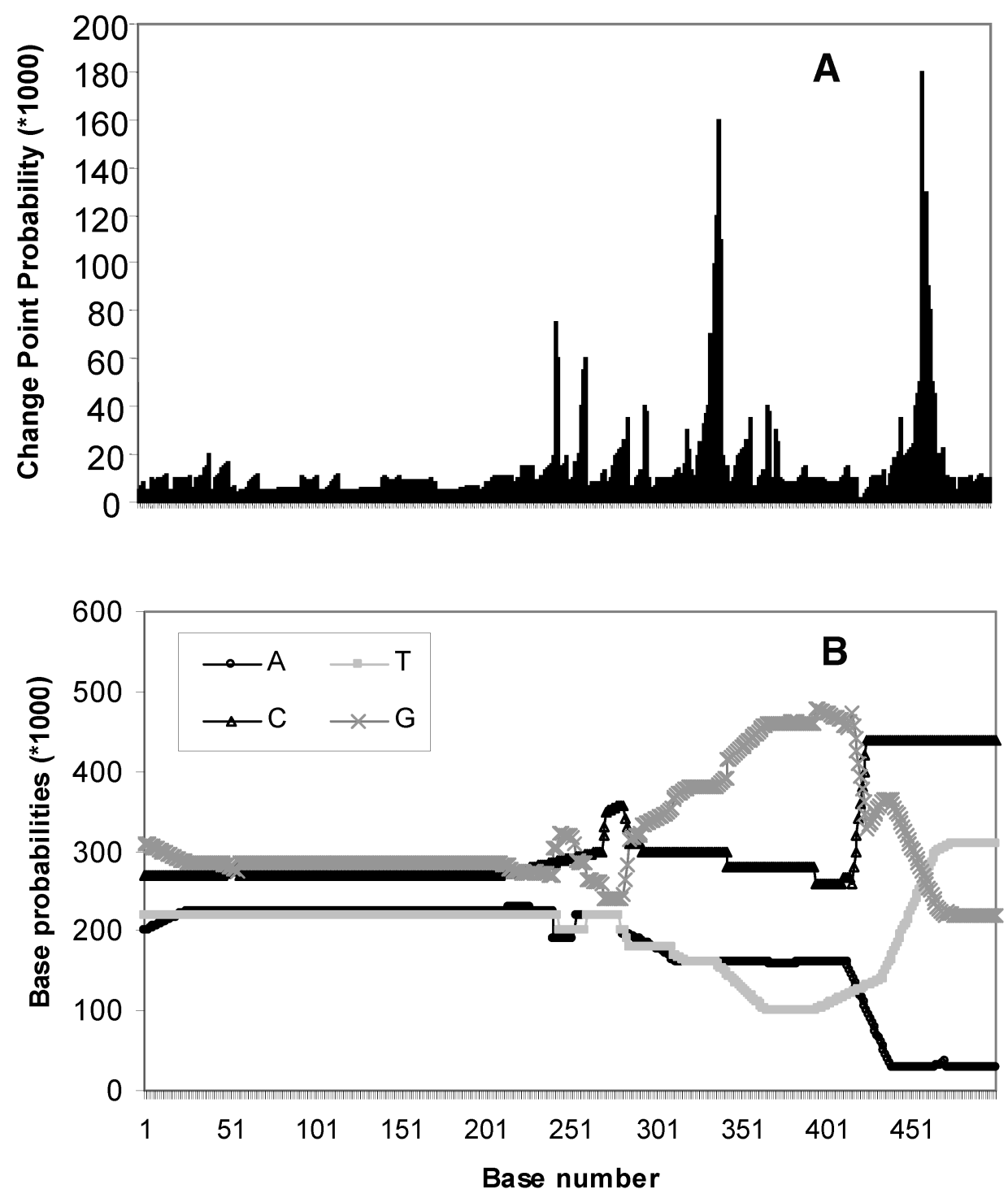

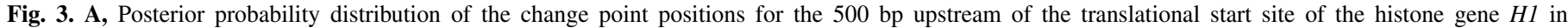

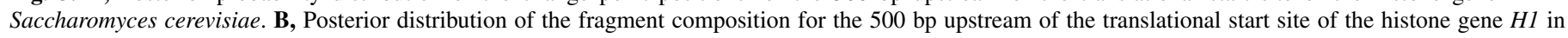
Saccharomyces cerevisiae. (reproduced, with permission, from Liu and Lawrence [7]; copyright Oxford University Press). 
The third level of the hierarchy includes the prior distributions of $\beta, V_{j}$, and $U_{j}$. Commonly, the unstructured residual is assumed to have a prior distribution, $V_{j} \sim$ iid. $N\left(0, \sigma_{v}{ }^{2}\right)$, and the spatially dependent residual is often assumed to have a multivariate normal distribution, $N\left(0, \sigma_{u}^{2} \sum(\varphi)\right)$. Here, $\Sigma(\varphi)$ is a correlation matrix with diagonal elements equal to one and off-diagonal elements representing the correlation between $U_{i}$ and $U_{j}, i \neq j$ (8).

The map of fitted asthma rates in each of the 1983 zip codes that was obtained using a model like the one described above is given in Figure 5B. The figure shows the characteristic Bayesian "smoothing" of the crude rates. In particular, few zip codes are now assigned a rate of zero, and the rather high rates in the thinly populated eastern part of the map have been substantially reduced (12). Some of the clusters of similar crude rates are preserved and the high observed rates in urban San Diego continue to be high (12). The model just described is used frequently when mapping is performed for decision-making on the distribution of public health benefits or environmental justice. Similarly, it could offer more insight in regional-scale risk assessment of plant diseases.

Update current knowledge as new data become available. When an experiment is designed, decisions are made before data collection, and data collection is often restricted by limited resources. Decisions that must be made by the scientist include the choice of treatments, blocking factors, experimental units, sample size, and randomization mechanism. That means that experimental results depend on the experimental design and, ultimately, on the decision made about which experimental design should be followed to collect the sample data (3). How can we be sure that we made the best possible decision regarding the design of our experiment? To answer this question, we need to use the utility function $U$ (a measurement of satisfaction from a decision). Lindley (6) suggests using a utility function that describes the purpose of the experiment well and then selecting the design that maximizes this utility.

Frequently, plant pathologists are interested in monitoring the development of resistance of plant pathogens to commercial pesticides. Typically, a first step in this procedure is the establishment of baseline sensitivity: determining the sensitivity to the pesticide using pathogenic isolates never treated with the com-
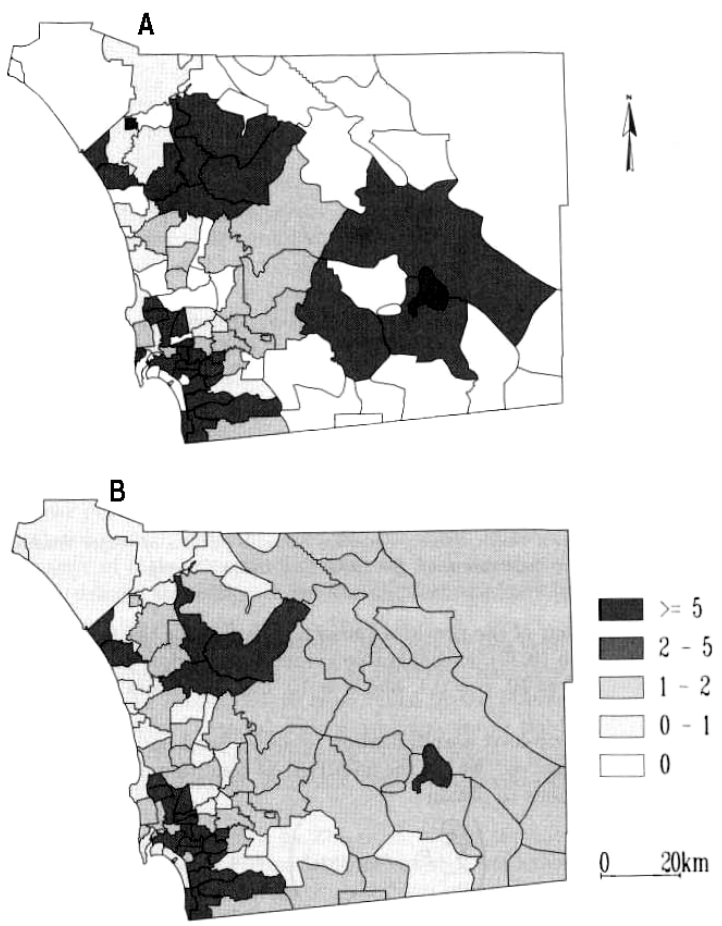

Fig. 5. Pediatric asthma hospitalization rate (per 1,000 children) by zip code for 1983, San Diego County, CA: A, crude rates and B, model fitted rates. (reproduced, with permission, from Zhu et al. [12]; copyright John Wiley \& Sons). pound. Assume that 200 fungal isolates, collected from fields where the pesticide was never applied, were used to determine the $\mathrm{EC}_{50}$, the concentration that results in $50 \%$ reduction in radial growth of the fungus. Monospore cultures of the isolates were plated in six concentrations of the pesticide $(0,1,2,3,4$, and $5 \mu \mathrm{g}$ $\mathrm{ml}^{-1}$ ). Radial growth of germinated spores was measured after 7 to 10 days in incubation to determine $\mathrm{EC}_{50}$.

There are two alternatives to determining the baseline sensitivity: (i) use the 200 isolates simultaneously and determine the baseline sensitivity $\left(\mu \mathrm{g} \mathrm{ml}^{-1}\right)$ based on the frequencies of $\mathrm{EC}_{50}$ values or (ii) use, initially, a subgroup of the 200 isolates with the concentrations mentioned above. Assume that from testing the subgroup the baseline sensitivity was calculated to be between 3.2 and $4.2 \mu \mathrm{g} \mathrm{ml}^{-1}$. Then we could use the $\mathrm{EC}_{50}$ calculated from the tested subgroup to construct a prior distribution for the subsequent test of the remaining isolates. For example, the sensitivity values might be assumed to have been drawn from a normal distribution with mean 3.7 and variance 0.5 (3). With such a prior distribution, we may conclude that for the rest of the isolates the concentrations to be used should be $3.07,3.47,3.73,4.13$, and 4.3 , different from the equally spaced original doses used in the original experiment.

Which alternative should we follow? The answer depends on the purpose of the experiment. Sometimes baseline sensitivity needs to be estimated with high precision because even slight changes in resistance could be important to a management program. If the purpose is to define baseline sensitivity with high precision, the second alternative might serve this purpose better than the first one. In this case, the utility function for the two alternative designs may be used as a formal rule to decide which alternative should be followed (3).

Software availability. Many of the algorithms for performing Bayesian analysis are available online. Most of them are contained in StatLib, a system for the electronic distribution of software, data sets, and other information that can be assessed via the website of the Statistics Department at Carnegie Mellon University. The most well-developed and widely used software for Bayesian analysis is BUGS (Bayesian inference using Gibbs sampler) available online from the Biostatistics Unit (BSU) of the Medical Research Council of Cambridge, UK $(9,10)$.

\section{LITERATURE CITED}

1. Box, G. E. 1980. Sampling and Bayes' inference in scientific modeling and robustness. J. R. Statist. Soc. A 143:383-430.

2. Carlin, B. P., and Louis, T. A. 2000. Bayes and Empirical Bayes Methods for Data Analysis. Chapman \& Hall/CRC Press, Boca Raton, FL.

3. Chaloner, K., and Verdinelli, I. 1996. Bayesian experimental design: A review. Statist. Sci. 10:273-304.

4. Gelman, A., Carlin, J. B., Stern, H. S., and Rubin, D. B. 1996. Bayesian Data Analysis. Chapman \& Hall/CRC Press, Boca Raton, FL.

5. Lawson, A. B. 2001. Tutorial in biostatistics: Disease map reconstruction. Statist. Medic. 20:2183-2204.

6. Lindley, D. V. 1990. The 1988 Wald Memorial Lectures: The present position in Bayesian statistics. Statist. Sci. 5:44-65.

7. Liu, J. S., and Lawrence, C. E. 1999. Bayesian inference on biopolymer models. Bioinformatics 15:38-52.

8. Pascutto, C., Wakefield, J. C., Best, N. G., Richardson, S., Bernardinelli, L., Staines, A., and Elliott, P. 2000. Statistical issues in the analysis of disease mapping data. Statist. Medic. 19:2493-2519.

9. Spriegelhalter, D. J., Thomas, A., Best, N., and Gilks, W. R. 1995. BUGS: Bayesian inference using Gibbs sampling, Version 0.50. Technical Report, Medical Research Council Biostatistics Unit, Institute of Public Health, Cambridge University, UK.

10. Spriegelhalter, D. J., Thomas, A., Best, N., and Gilks, W. R. 1995. BUGS Examples, Version 0.50. Technical Report, Medical Research Council Biostatistics Unit, Institute of Public Health, Cambridge University, UK.

11. Townsend, J. P., and Hartl, D. L. 2002. Bayesian analysis of gene expression levels: Statistical quantification of relative mRNA level across multiple strains or treatments. Gen. Biol. 3:1-16.

12. Zhu, L., Carlin, B. P., English, P., and Scalf, R. 2000. Hierarchical modeling of spatio-temporally misaligned data: Relating traffic density to pediatric asthma hospitalizations. Environmetrics 11:43-61. 